\title{
The Challenge of Making Augmented Reality Work Outdoors
}

\author{
Ronald T. Azuma \\ HRL Laboratories \\ 3011 Malibu Canyon Road MS RL96; Malibu, CA 90265; USA \\ Office: (310) 317-5151; Fax: (310) 317-5695 \\ a zuma@HRL . com
}

\section{Background in Augmented Reality}

Making Augmented Reality (AR) systems that work outdoors is a natural step in the development of AR toward the ultimate goal of AR displays that can operate anywhere, in any environment. To place this in context, this section gives a brief overview of the development of AR, my own contributions to this field, and our current research program at HRL Laboratories.

Despite its potential, Augmented Reality has not received nearly the amount of attention paid to its sibling, Virtual Environments (or Virtual Reality), despite the fact that both fields share a common ancestor. It is often forgotten that Sutherland's original HMD system was an optical see-through display. While the creators of that system did not explicitly attempt to register virtual 3-D objects with real-world objects, they did have an example of combining virtual and real. The motivation was to allow the user to issue commands. The problem was that the graphics engine did not have sufficient power to draw the menus and commands virtually at interactive rates. Therefore, they physically put large signs with the command names on a real wall, and allowed the user to virtually select one of the real signs by pointing at one with the hand controller. Despite this common root, most efforts following Sutherland's focused on Virtual Environments. It wasn't until the late 1980's and early 1990's that research in Augmented Reality began again in earnest.

While there are several problems in building Augmented Reality systems, many research efforts have focused on the registration problem (the proper alignment of virtual with real). This is a difficult problem because there are many sources of error, even small amounts of error are perceptible, and few commercially-available trackers provide the required performance. Without good registration, many AR applications will not be accepted. My own research history tackled this area. I contributed to a wide-area optical tracking system for HMDs [Ward92] [Azuma93], and then built and analyzed an optical see-through HMD system that used head-motion prediction and other techniques to greatly reduce registration errors [Azuma94] [Azuma95]. I also wrote a survey paper [Azuma97] that summarizes the work done in this field.

What is the current state-of-the-art in registration today? A few prototype systems have demonstrated almost pixel-accurate displays for certain applications. These typically use a "closed loop" tracking approach [Bajura95], where the real-world images shown in a video see-through display are also used to aid the tracking, correcting remaining errors to ensure that the virtual is properly aligned with the real objects captured in the video images. A good example of this is [State96]. Not every AR application requires pixel-accurate registration, and other effective demonstrations and prototype systems have been built for manufacturing and maintenance of aircraft parts [Nash97] and even for entertainment [Ohshima98]. Therefore, the current state-of-the-art is that effective prototype systems exist for a few applications, and at least one is getting close to actual deployment on a factory floor. 
While there has been significant progress on the registration problem, it is still far from a solved problem. AR systems, especially those that rely on closed-loop tracking, required carefully controlled environments. The user cannot walk and look anywhere she pleases; the system only works for specific objects, as seen from a limited range of viewpoints. By constraining the problem in this manner, the registration problem is more tractable, but it also greatly restricts the flexibility of the system and makes it difficult to build an effective AR system without expert knowledge. A symptom of this is the unavailability of commercial systems. Today, one has a choice of several turnkey Virtual Environment systems, but nobody offers a turnkey Augmented Reality system. Contrast the situation today with the ideal situation: where one can put on an AR display and walk around anywhere, with no restrictions. Ideally, an AR display should work in all environments without the need to prepare rooms and objects ahead of time. There is a need to investigate AR systems that can work in unstructured, real-world environments, both indoors and outdoors. Some work along this direction has been done to reduce the calibration requirements [Kutukalos98] and the need to know the geometry of all observed objects at the start [Iu96]. However, to continue making progress, we need to explore more difficult problem domains. We should explore AR in outdoor applications, rather than just the indoor applications that have dominated the field so far.

Exploring outdoor AR systems and applications is the current research direction in AR for personnel at HRL Laboratories. Funding is provided by a DARPA grant for a project called GRIDS: Geospatial Registration of Information for Dismounted Soldiers. The primary personnel involved with the project at HRL are myself, Bruce Hoff, Howard Neely, Ron Sarfaty, and Mike Daily. We are collaborating on this research with our partners at the University of North Carolina at Chapel Hill (Gary Bishop, Vern Chi, and Greg Welch), the University of Southern California (Ulrich Neumann and Suya You), and Raytheon Systems Company (Rich Nichols and Jim Cannon). While the initial users this project is focusing on are infantrymen, this technology will also be suited for a variety of civilian users and applications. This chapter outlines the motivations and potential applications of AR in outdoor situations (Section 2), analyzes the problems we face in building outdoor AR systems, especially in the area of tracking (Section 3), and concludes by suggesting some general approaches (Section 4).

\section{Motivation for Outdoor Augmented Reality}

Augmented Reality systems that provide accurate registration outdoors are of interest because they would make possible new application areas and could provide a natural interface for wearable computers, an area of growing interest both in academia and industry. A user walking outdoors could see spatially located information directly displayed upon her view of the environment, helping her to navigate and identify features of interest. Today, a hiker in the woods needs to pull out a map, compass, and GPS receiver, convert the GPS and compass readings to her location and orientation, and then mentally align the information from the 2-D map onto what she sees in the 3-D environment around her. A personal, outdoor AR system could perform the same task automatically and display the trail path and landmark locations directly upon her view of the surrounding area, without the cognitive load. Soldiers could see the locations of enemies, friends, and dangerous areas like minefields that may not be readily apparent to the naked eye.

Personal, outdoor AR systems would also be useful for groups of users working together. If the users are widely separated, it is difficult for them to establish common frames of reference to describe spatially located information. An instruction telling another team member to go to the "3rd white building to the left of the red building" may be useless if the listener sees the world from a different vantage point than the speaker. Personal AR 
displays provide an unambiguous method of sharing such information. Furthermore, personal outdoor AR displays may be a natural interface for wearable PC's. The standard WIMP interface does not map well onto wearable PCs, because the desktop metaphor is not appropriate for a user walking around outdoors who may not have her hands free or be able to allocate complete attention to the computer [Rhodes98]. Augmented Reality may be a better approach for certain applications.

Another application area is the visualization of locations and events as they were in the past or as they will be after future changes are performed. Tourists that visit historical sites, such as a U.S. Civil War battlefield or the Acropolis in Athens, Greece, do not see these locations as they were in the past, due to changes over time. It is often difficult for a modern visitor to imagine what these sites really looked like in the past. To help, some historical sites stage "Living History" events where volunteers wear clothes of that time period and reenact historical events. A tourist equipped with an outdoor AR system could see a computer-generated version of Living History. The HMD could cover up modern buildings and monuments in the background and show, directly on the grounds at Gettysburg, where the Union and Confederate troops were at the fateful moment of Pickett's charge. The gutted interior of the modern Parthenon would be filled in by computer-generated representations of what it looked like in $430 \mathrm{BC}$, including the longvanished gold statue of Athena in the middle. Tourists and students walking around the grounds with such AR displays would gain a much better understanding of these historical sites and the important events that took place there. Similarly, AR displays could show what proposed architectural changes would look like before they are carried out. An urban designer could show clients and politicians what a new building would look like as they walked around the adjoining neighborhood, to better understand how that skyscraper might affect nearby residents.

If truly effective outdoor AR displays existed, then some applications that we normally view as indoor applications may find new roles in outdoor situations. The maintenance and repair of vehicles in the field would be helpful for users stuck far away from traditional repair facilities. Aircraft technicians could use such displays when working on aircraft outdoors, at terminals. Paramedics might combine such displays with portable non-invasive 3-D scanners to provide a "3-D stethoscope" in the field. And the possibilities AR has for outdoor entertainment applications are unexplored.

While outdoor AR applications offer some interesting possibilities, few have attempted to build Augmented Reality systems that work outdoors. Steve Feiner's group at Columbia demonstrated the Touring Machine, which allows a user to view information linked to specific buildings on the Columbia campus as he walks around outside [Feiner97]. Some wearable computers, such as the CMU VuMan systems, have been used for vehicle maintenance applications in outdoor settings. However, none of these previous works focused on achieving accurate registration at a wide variety of outdoor locations, and to my knowledge, none offers the performance required for most outdoor applications.

\section{Analysis of Problem Areas}

What are the difficulties that prevent personal, outdoor AR systems from being deployed today? The ergonomic issues that face wearable PC systems apply to outdoor AR systems as well: size, weight, power, ruggedness, etc. Displays that have sufficient contrast to work in outdoor settings are required. But the biggest challenge lies in accurate tracking outdoors: determining the user's position and orientation with sufficient accuracy to avoid significant registration errors. This section analyzes these three problem areas. 


\subsection{Size, Weight and Power Issues}

A major difference between outdoor and indoor AR systems is the amount of infrastructure and resources available. Indoor systems work in relatively luxurious conditions. Power, computation, and other resources are limited by the agency's budget rather than ergonomic constraints. An indoor application must worry about what the user wears on his head, but the user need not carry the computers or the power supplies. An extensive infrastructure can be available indoors, with a wider range of tracking technologies that may not be practical outdoors. The situation changes dramatically once you go outdoors. If the user is in a vehicle, then the computation and power budget may be relatively large. But if the user walks around outdoors on his own and must carry batteries, the computer, and all other resources, then size, weight and power issues become significant concerns.

The amount of gear that a user can be reasonably asked to carry depends on the type of person and the application. Helmets for infantrymen and military pilots typically weigh around four to five pounds. For civilian applications that support a wider user base than the military, the weight limit is probably far less. Minimizing weight on the head is more critical than minimizing weight in the backpack or belt, but overall weight is a concern as well. Developments in laptops, wearable PC's, PDA's and other upcoming portable devices may provide improvements that outdoor AR systems can leverage off of.

\subsection{Displays}

For indoor applications, we can control the lighting to match the display. But in outdoor applications, the AR display must work across a wide variety of lighting conditions, from bright sunlight to a moonless night. The contrast between these two conditions is huge, and most display devices cannot come close to the brightness required to match this range. This is no surprise to the laptop owner who tries to read his display in bright sunlight. For optical see-through displays this can be a big problem because the user sees the real world directly through the display. Certain upcoming displays, such as FEDbased devices, may be bright enough to be visible in bright sunlight. But for the rest, it will be necessary to provide shading devices to cut down on the amount of light entering the display (much like a pair of sunglasses). Video see-through avoids some of this problem because one can set the exposure of the cameras to match the input. However, this comes at a price. The dynamic range of the real world must be compressed into the range of the output monitor, which is generally much smaller. Also, the resolution of the display monitor is much lower than the resolution of the human eye. These two combined will cause a loss of detail that may not be acceptable in some applications.

A primary motivation for using video see-through is that it allows closed-loop tracking approaches. Since the opportunity to do closed-loop tracking is reduced when we cannot modify the environment and place fiducial markers at known locations, the desirability of video see-through displays may be lower in outdoor applications. Video see-through also demands greater amounts of computation power and other resources, which are limited in outdoor applications.

\subsection{Tracking}

Accurate tracking indoors is hard enough; accurate tracking outdoors is even more daunting because of two main differences in the situation. First, we have less control over 
the environment. Second, we have fewer resources available -- power, computation, sensors, etc. These differences mean that solutions for indoor AR may not directly apply to personal outdoor AR systems. For example, several indoor AR systems have achieved accurate tracking and registration by carefully measuring the objects in a highly constrained environment, putting colored dots over those objects, and tracking the dots with a video camera (see [Azuma97] for some references). This approach violates some of the constraints for an outdoor situation. We do not have control over the outdoor environment and cannot always rely on modifying it to fit the needs of the system. For example, in a military application it is not realistic to ask soldiers, friendly or enemy, to wear large, brightly colored dots to aid our tracking system. We should not expect to measure every object in the environment beforehand. Also, this approach may require more computational resources than is practical for a single outdoor user. Such systems have used an SGI Onyx or other high-end workstation with frame-grabbing capability and sufficient bandwidth to process captured frames at interactive rates.

Initial outdoor AR systems may get by with less accurate registration because some outdoor applications may have less stringent requirements than their indoor counterparts. A doctor performing a needle biopsy requires that the virtual incision marker be accurate within a millimeter, but for a hiker walking around, perhaps even one degree of angular error is acceptable to cue the user to landmarks in the environment. However, the ultimate goal is still pixel-accurate registration under all circumstances.

We can analyze this problem by examining the various individual tracking technologies that are available outdoors. This will include a closer examination of some data taken from a sourceless outdoor orientation sensor.

- GPS: The Global Positioning System provides worldwide coverage and measures the user's 3-D position typically within 30 meters for regular GPS and about 3 meters for differential. It does not directly measure orientation. Differential accuracy is sufficient for viewing distant but not nearby objects; at 50 meters range a 3 meter position error results in 3.4 degrees of registration error. A typical update rate from a differential GPS receiver is 1 $\mathrm{Hz}$, although I have seen some that claim $10 \mathrm{~Hz}$. Again, this is sufficient for distant objects but not for nearby objects. For some vehicles, one can use several GPS receivers and establish sufficient baselines to recover vehicle orientation. But for a user walking around, that's not practical. The baseline required is larger than a user can be reasonably asked to support. New carrier-phase GPS receivers claim accuracy in centimeters. However, achieving such accuracies at interactive rates will be difficult due to multipath problems; avoiding those requires that the antenna be the highest object in the surrounding environment, which clearly is not practical under many circumstances [Bishop98] [Kaplan96]. The startup delay of a GPS unit can vary from several seconds to several minutes, depending on whether or not the unit has a good initial guess of the current location and time. GPS requires direct line-of-sight to a sufficient number of satellites, so it works best in open areas. When inside urban areas, canyons, near hills and other terrain, GPS is often blocked. In military situations, GPS is easily jammed.

- Inertial and dead reckoning: Inertial sensors are sourceless and relatively immune to environmental disturbances. Their main problem is drift: the accumulation of error with time. Recovering orientation changes (through rate gyroscopes) is easier than position changes (through linear accelerometers) because the former requires only one integration step, while the latter requires two. Ships and aircraft have inertial measurement units that drift a fraction of a degree per hour in orientation and under one nautical mile per hour in position. However, these sensors are not appropriate for a single user walking around outdoors, due to cost and weight. Rate gyros of the form factor appropriate for a human exist and may be sufficient for shorter time intervals (minutes or seconds). However, no 
accelerometers exist that can provide the performance desired (millimeters of drift over long time periods). This requires accelerometers with bias accuracies several orders of magnitude better than what is commonly available today. Pedometers or other dead reckoning trackers may be better solutions for the individual user walking around.

- Active sources: Setting up active transmitters and receivers (using magnetic, optical, or ultrasonic technologies) is commonly done for indoor Virtual Environment systems. Modifying the environment in this manner outdoors may be feasible for a few applications, such as an outdoor maintenance application. However, this approach tethers the user a particular area outdoors (close to the active sources) and requires an infrastructure (power, supporting structures to hold sources in position, etc.) that may not be easy to construct outdoors. For many outdoor applications, such as hiking in the woods, it is not practical to modify the environment in this manner, especially given the user's range of operation.

- Passive optical: Using video sensors to track the user location based upon what is visible (the sun, the stars, and the view of the surrounding environment) provides the ability to do closed-loop tracking approaches. Video sensors are line-of-sight and will lose lock if the view is obscured (by buildings, vegetation, etc.) Computer vision techniques are not currently robust enough to provide a complete sole solution, and unless known landmarks are used, the tracking solutions are relative rather than absolute. Tracking based on real-world features and scenes is also more difficult and yields more brittle results than closed-loop tracking of fiducials that some indoor AR systems use. Video processing is also computationally intensive and is not feasible in real time on most wearable PC systems.

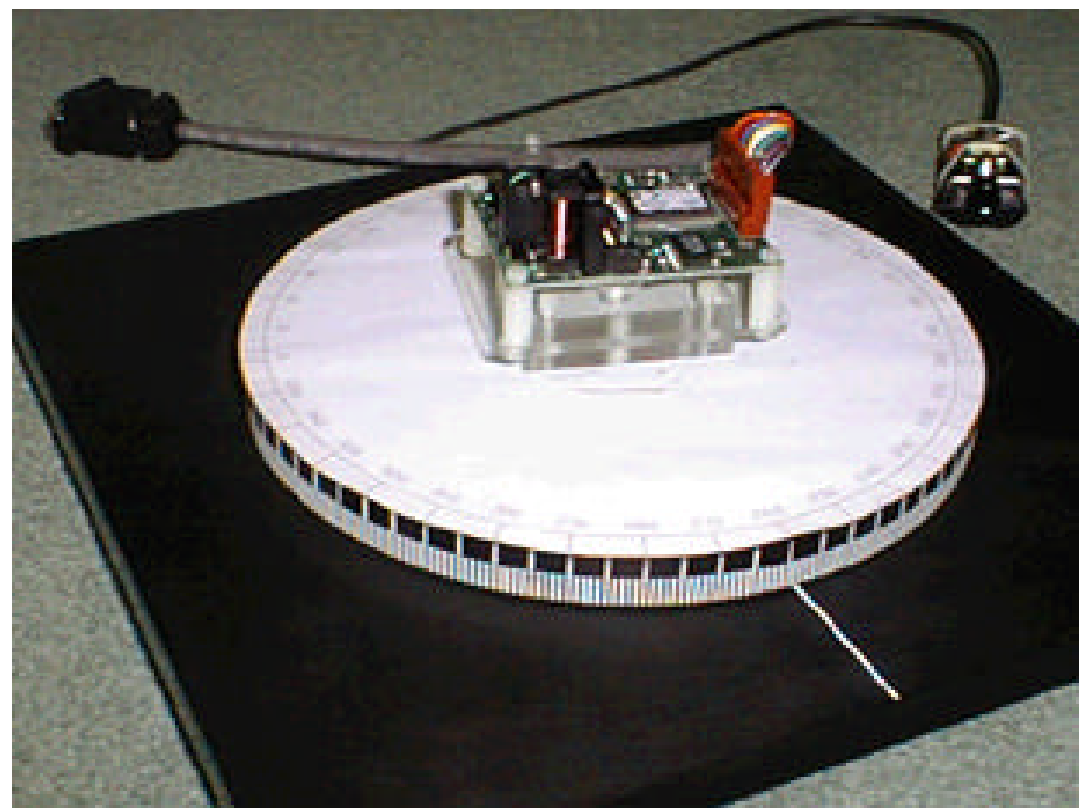

Figure 1: Turntable for measuring distortion in electronic compass (Precision Navigation TCM2). Turntable is made of Delrin to avoid creating an additional source of distortion.

- Electronic compass and tilt sensors: One of the most common trackers in Virtual Environments is used by inexpensive HMD's: a sourceless orientation-only head tracker that consists of an electronic compass and two tilt sensors. These trackers can be small and inexpensive. The best units claim a yaw accuracy of \pm 0.5 degrees, with smaller errors along roll and pitch. Such a sensor is attractive because it can potentially provide 
orientation measurements without the need of any supporting infrastructure. We have experimented with one such sensor, the Precision Navigation TCM2. The update rate is approximately $16 \mathrm{~Hz}$. The main limitations we have encountered are the distortion, noise and delays in the sensor output.

The compass is highly sensitive to disturbances in the Earth's magnetic field. In hindsight, this is not surprising, because Earth's magnetic field is a relatively weak signal. Figure 1 shows the equipment we used to measure distortions in the field. The mechanical turntable is made of Delrin and other non-ferrous materials, to avoid placing sources of distortion near the compass. We measured the compass output with this device at many different locations and times. The compass was sampled at 5 degree intervals. Even when the compass was far from any apparent sources of distortion, the compass measurements would be in error by up to 3 degrees, and the distortion pattern varies significantly with time and location, as shown in Figure 2. These are for measurements taken in "magnetically clean" environments, far from any known sources of distortion. However, in realistic AR systems, it is difficult to completely avoid all sources of magnetic distortion. In a more realistic situation, the peak-to-peak distortion errors were typically 20-30 degrees. The distortion makes it difficult to use such a sensor in an AR system without calibration or other compensation strategies. Because some of the curves in Figure 2 have radically different shapes than the others, developing adequate models that compensate for this distortion is a significant challenge. In other words, the magnetic distortion appears to be significantly different at different geographical locations, and even at different times at the same location.

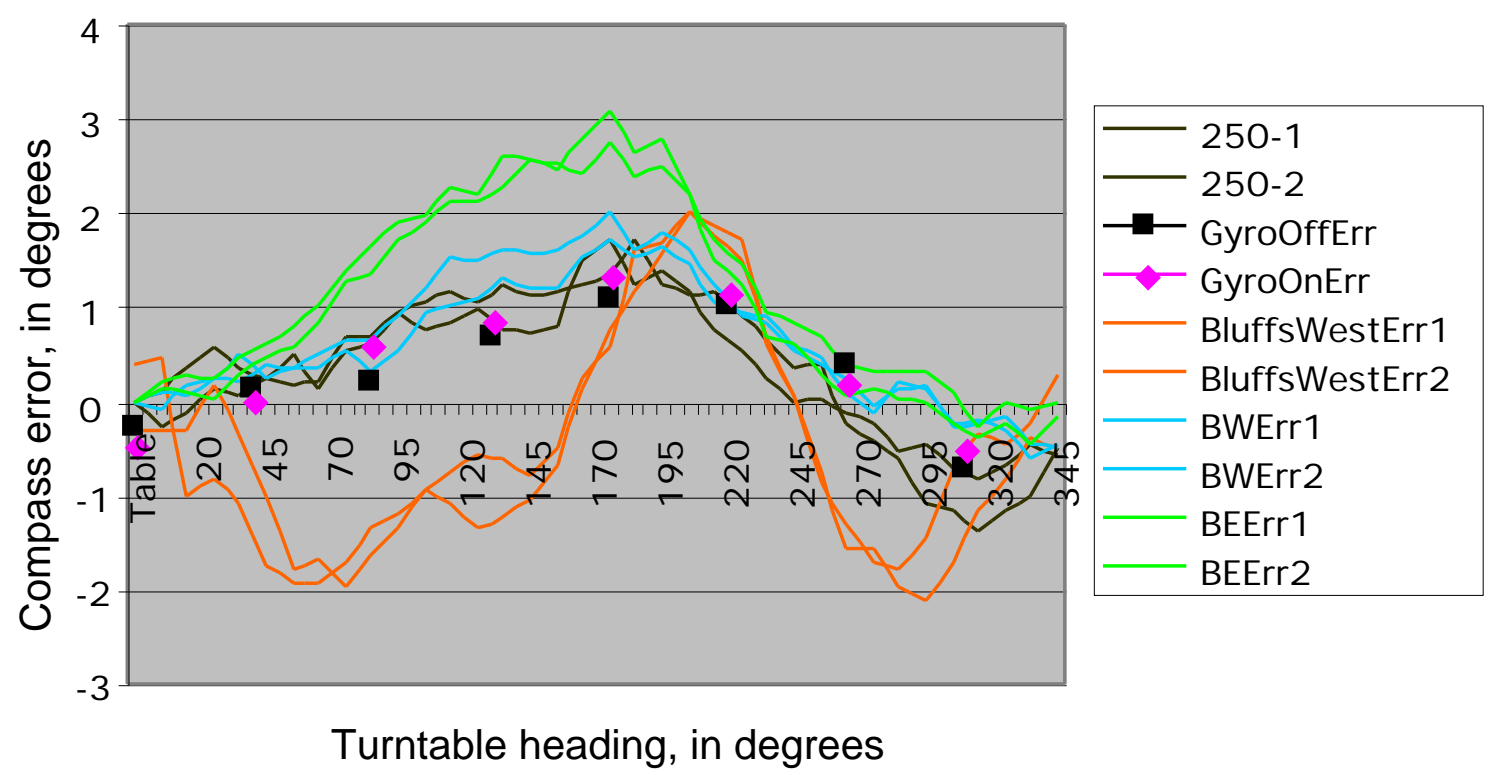

Figure 2: Distortion in TCM2. Data taken on turntable, shown in Figure 1. $\mathrm{X}$ axis is yaw orientation of compass in degrees, $\mathrm{Y}$ axis is the relative error in degrees from the zero degree initial orientation.

The TCM2's output is noisy, both when sitting still and when the user moves around. When the sensor sits still, the output will typically have noise around 0.5 degrees, which is quite visible inside an AR display. Recall that the apparent width of the full moon is 0.5 degrees. When the TCM2 is moved around, the noise becomes unbearably large, on the order of several degrees. This is partially due to the "sloshing" of the inclinometers when in motion. Translation motion will change the measured orientation outputs, even if 
the true orientation has not changed. This is a basic limitation of using tilt sensors. Even the small motions imparted by a casual walk or by gusty winds are enough to render the output inaccurate. If one expects to use this sensor while walking around or riding in a vehicle, then the AR system must compensate for these noise sources.

Finally, the TCM2's output is delayed in time by $\sim 100$ milliseconds. This is due to the settling time of the inclinometers (which affect the computed yaw reading from the compass) and the use of a serial line as the communication link, which is relatively slow. Sensor lags of this magnitude are serious in AR applications because system delay is the single largest contributor to registration error in most systems [Holloway97].

Some of these problems might be reduced by switching to a different compass and tilt orientation sensor, but the serious ones are endemic to any sensor using this technology. The magnetic distortion is different at different locations on the ground; more accurate sensors will not change that fact. And the distortion introduced when moving around will occur in any tilt-based sensor. While sourceless orientation sensors of this type are an interesting starting point for outdoor AR tracking, by themselves they will not be sufficient to achieve our ultimate goals.

\section{Approaches and Conclusions}

From this analysis of the tracking technologies available outdoors, we find that no single technology provides a complete solution. In the near term, no single technology is projected to provide the performance we require to meet our goals. Therefore, combining several tracking technologies, or hybrid tracking, is the only feasible approach for the near term. Hybrid approaches increase system complexity and cost but provide the most robust results. Hybrid approaches allow the weaknesses in each individual technology to be covered by some other sensor, resulting in an overall system that behaves more robustly than with each sensor applied individually.

What areas of outdoor tracking are most critical to attack initially? To answer that, we examine the contributions to registration error. Let's take a simple example, using a system similar to [Feiner97] as the current state-of-the-art for personal outdoor AR tracking. That system measures position by a differential GPS and orientation by a compass and tilt sensor similar in performance to the one discussed in Section 3.3. Assume that the real-world object being viewed is 100 meters away, and that the user rotates his head at a moderate rate of 50 degrees per second. Note that even with a heavy HMD, we have measured peak head rotation rates around 80 - 120 degrees per second, and with minimal encumbrance on the head, peak rotation rates can exceed 300 degrees per second, so the assumption of 50 degrees per second is conservative. Assume the sensor itself has 100 milliseconds of delay internally, and the rest of the system adds another 100 milliseconds (for $200 \mathrm{~ms}$ total). The peak error of the differential GPS measurement is 3 meters, and the peak error of the compass is 3 degrees. Then the expected contributions to registration error are as shown in Table 1:

\begin{tabular}{|c|c|}
\hline Contribution to & Range of error \\
registration error & $0-1.7$ degrees \\
\hline $\begin{array}{c}\text { Position errors } \\
\text { Static orientation } \\
\text { errors }\end{array}$ & $0-3$ degrees \\
$\begin{array}{c}\text { Dynamic orientation } \\
\text { errors }\end{array}$ & 10 degrees \\
\hline
\end{tabular}


Table 1: Quantifying contributions to registration error

Clearly, the largest individual contributor is the dynamic orientation error, thus that is the most important one to tackle initially. This source can be attacked by reducing all sources of latency within the system and compensating for what remains via predictive means. However, the other two sources are also much larger than desired, so those must not be ignored either.

Although accurate tracking outdoors is a difficult problem, it is a worthwhile challenge because developing new tracking technologies that work in an outdoor realm will lead to improvements for AR and Virtual Environment systems overall. Research in this direction will help push AR systems away from the highly specialized, difficult-to-build prototype systems of today to more flexible, portable systems that can be deployed anywhere. I believe improvements in such areas must be made if AR systems are to appear commercially in wide numbers, and I hope that they will enable the applications described earlier in this paper, along with others we have yet to imagine.

\section{Acknowledgments}

Our work in this area is mostly funded by DARPA contract N00019-97-C-2013, from the DARPA ETO Warfighter Visualization program.

\section{References}

Azuma93

Azuma94

Azuma95

Azuma97

Bajura95

Bishop98

Feiner97
Azuma, Ronald. Tracking Requirements for Augmented Reality. Communications of the ACM 36, 7 (July 1993), 50-51.

Azuma, Ronald and Gary Bishop. Improving Static and Dynamic Registration in an Optical See-Through HMD. Proceedings of SIGGRAPH '94 (Orlando, FL, 24-29 July 1994), Computer Graphics Annual Conference Series, 1994, 197-204.

Azuma, Ronald and Gary Bishop. A Frequency-Domain Analysis of Head-Motion Prediction. Proceedings of SIGGRAPH '95 (Los Angeles, CA, 6-11 August 1995), Computer Graphics Annual Conference Series, 1995, 401-408.

Azuma, Ronald T. A Survey of Augmented Reality. Presence: Teleoperators and Virtual Environments 6, 4 (August 1997), 355385.

Bajura, Michael and Ulrich Neumann. Dynamic Registration Correction in Video-Based Augmented Reality Systems. IEEE Computer Graphics and Applications 15, 5 (September 1995), 5260.

Bishop, Gary. Personal communication (January 30, 1998).

Feiner, Steven, Blair MacIntyre, and Tobias Höllerer. A Touring Machine: Prototyping 3D Mobile Augmented Reality Systems for Exploring the Urban Environment. Proceedings of First 
International Symposium on Wearable Computers (Cambridge, MA, 13-14 October 1997), 74-81.

Holloway97 Holloway, Richard. Registration Error Analysis for Augmented Reality. Presence: Teleoperators and Virtual Environments 6, 4 (August 1997), 413-432.

Iu96

Iu, Siu-Leong and Kevin W. Rogovin. Registering Perspective Contours with 3-D Objects Without Correspondence Using Orthogonal Polynomials. Proceedings of VRAIS '96 (Santa Clara, CA, 30 March - 3 April 1996), 37-44.

Kaplan96

Kaplan, Elliot D. (ed.) Understanding GPS Principles and Applications. Artech House Publishers, ISBN 0-89006-793-7, 1996.

Kutukalos98 Kutukalos, Kiriakos N. and James R. Vallino. Calibration-Free Augmented Reality. IEEE Transactions on Visualization and Computer Graphics 4, 1 (January - March 1998), 1-20.

Nash97

Ohshima98

Nash, Jim. Wiring the Jet Set. Wired 5, 10 (October 1997), 128135 .

Ohshima, Toshikazu, Kiyohide Satoh, Hiroyuki Yamamoto and Hideyuki Tamura. AR ${ }^{2}$ Hockey: A Case Study of Collaborative Augmented Reality. Proceedings of IEEE VRAIS '98 (Atlanta, GA, 14-18 March 1998), 268-275.

Rhodes98 Rhodes, Bradley. WIMP Interface Considered Fatal. Position paper at IEEE VRAIS '98 Workshop on Interfaces for Wearable Computers (Atlanta, GA, 15 March 1998).

State96

State, Andrei, Mark A. Livingston, Gentaro Hirota, William F. Garrett, Mary C. Whitton, Henry Fuchs and Etta D. Pisano. Techniques for Augmented-Reality Systems: Realizing UltrasoundGuided Needle Biopsies. Proceedings of SIGGRAPH '96 (New Orleans, LA, 4-9 August 1996), 439-446.

Ward92 Ward, Mark, Ronald Azuma, Robert Bennett, Stefan Gottschalk, and Henry Fuchs. A Demonstrated Optical Tracker With Scalable Work Area for Head-Mounted Display Systems. Proceedings of 1992 Symposium on Interactive 3D Graphics (Cambridge, MA, 29 March - 1 April 1992), 43-52. 